\title{
Analysis of single frequency modulation for passive hydrogen maser
}

\author{
Hefei Zheng*, Jing Li, Wenming Wang, Lianshan Gao, and Keming Feng \\ Beijing Institute of Radio Metrology and Measurement, The Second Academy of China Aerospace Science and Industry Corporation, \\ Beijing 100854, China
}

\begin{abstract}
Based on the theory of the passive hydrogen maser, along with the technology of frequency modulation and modulation transfer spectroscopy, the theoretical expression of the single frequency modulation for the passive hydrogen maser and the function of the cavity and $\mathrm{H}$ line error signals separation are derived, which are basically coincident with the experiment. The absorption and dispersion spectrum curves with different resonance widths show that the cavity and hydrogen transition serve as discriminators, and the two error signals can be separated. Through the calculations of the two error signals in the passive hydrogen maser, it analyzes the traditional method of the two error signals separation, and then describes a new improved method for the passive hydrogen servo loops consisting in the use of a single modulation frequency and frequency discrimination. A null interaction of the two error signals for the new selection of the phase setting is deduced theoretically and validated by the simulation. The preliminary experimental result confirms the feasibility of this new approach, which can reduce the influence from the cavity frequency variety on the crystal oscillator and contribute significantly to the long term performance of the passive hydrogen maser.
\end{abstract}

Keywords: passive hydrogen maser, single frequency modulation, frequency discrimination.

DOI: $10.21629 / J S E E .2017 .04 .05$

\section{Introduction}

The passive hydrogen maser is one of the atomic standards with outstanding performance in terms of frequency stability and low drift [1,2]. Its realization comes as an extension of the attempt to reduce the volume and weight of the active hydrogen maser by reducing the microwave cavity dimensions, while the hydrogen atoms in the small size cavity need an excitation signal to stimulated emission [3]. The microwave cavity with wide bandwidth and the hydrogen transition with narrow linewidth act as discriminators, and the excitation signal is a microwave with

\footnotetext{
Manuscript received September 09, 2016.

*Corresponding author.

This work was supported by the Next Generation of Beidou Navigation Satellite (GFZX0301020104).
}

frequency modulation for frequency discrimination [4]. Therefore, the technology of frequency modulation is one of the key technologies for the passive hydrogen maser.

In the past, the servo control of the passive hydrogen maser used two modulation frequencies. The interrogation signal is modulated at two frequencies, one is a high frequency used to probe the cavity and the other is a low frequency used to probe the hydrogen resonance [5]. This signal, after going through the physical package, then contains amplitude modulation components at two frequencies, which lead to certain practical difficulties due to the interaction of the two control signals [6]. In order to overcome these problems, the passive hydrogen maser is based on the approach of single frequency modulation and phase discrimination. This approach requires a proper phase angle selection of the synchronous detectors at present in the voltage controlled crystal oscillator (VCXO) control loop and in the cavity control loop, in order to separate the two error signals [7]. The theoretical calculations of the single frequency modulation and separation of the two error signals are performed in this paper. The simulation results show the explanatory diagram and validate the calculations as well. Due to the interaction of the two error signals in the traditional method of phase selection, the component of the cavity error signal is in the VCXO control loop and influences the VCXO control voltage, which affects the accuracy of the passive hydrogen maser. Therefore, it is important to adopt a new method to separate the two error signals completely.

\section{Theory and calculation of single frequency modulation}

The idea behind the passive hydrogen maser is simple in principle: a frequency of VCXO is measured with the hyperfine transition of hydrogen atoms, and this measurement is fed back to the VCXO to suppress frequency fluctuations. The passive hydrogen maser includes two packages, the physical package serving as the discriminator 
and the electronic package producing frequency modulation signals and processing error signals [8,9]. Fig. 1 is a schematic diagram illustrating the two servo loops of the passive hydrogen maser. The interrogation signal after passing through the physical package includes the fre- quency information of the cavity and hydrogen line. It is analyzed by the envelope detector, the band-pass filter centered on the modulation frequency, the phase shifter and the integrator, and then controls the cavity frequency and the VCXO frequency respectively [10].

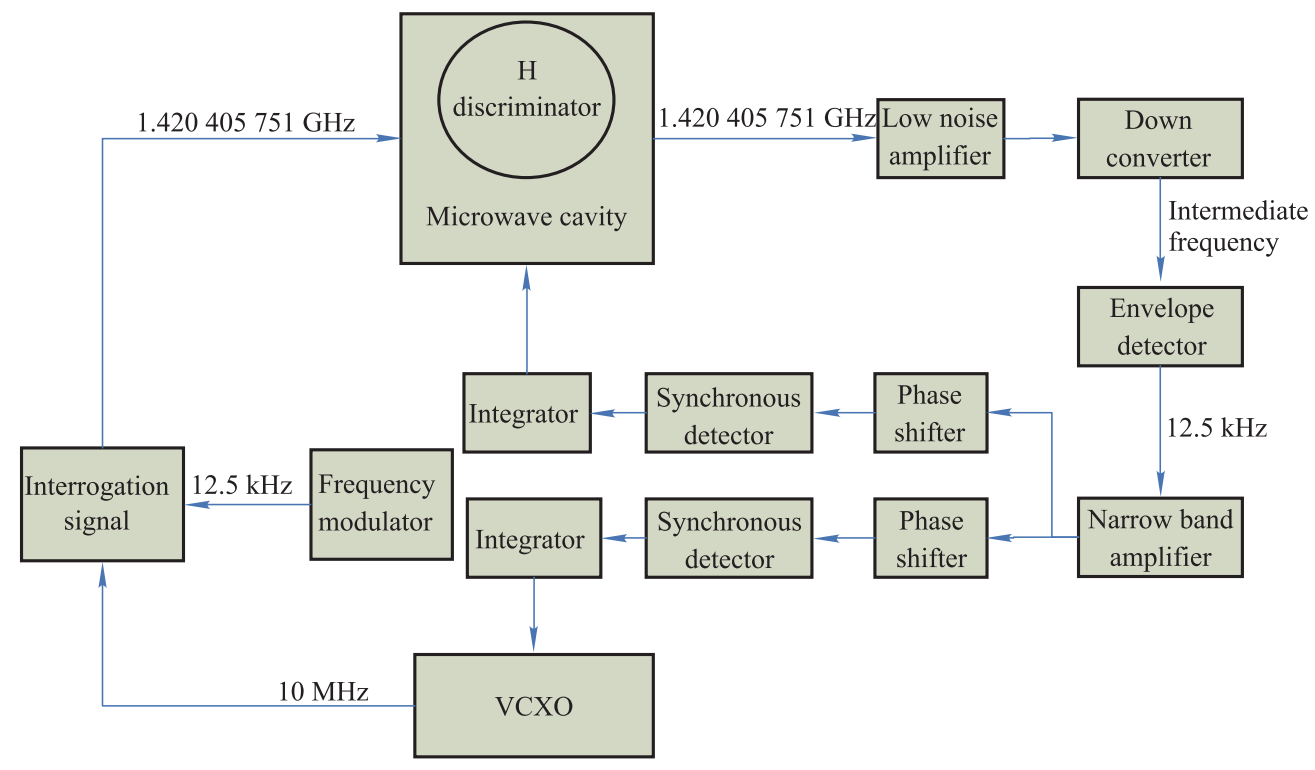

Fig. 1 Schematic diagram of the passive hydrogen maser

\subsection{Absorption and dispersion lock}

In the passive hydrogen maser, two error signals are obtained if the interrogation frequency differs from the hyperfine transition frequency and the cavity frequency, and are separated into two loops. The error signal with the frequency information between the hydrogen line and VCXO in the VCXO loop controls the VCXO, and the other error signal with the frequency information between the cavity and VCXO in the cavity loop tunes the cavity frequency in order to reduce the cavity pulling [11]. Due to the difference between the cavity bandwidth and the hydrogen transition linewidth, the modulation frequency is less than the cavity bandwidth and much greater than the hydrogen transition linewidth. For frequency locks, the cavity lock is called absorption lock, because the error signal results from the interaction of the carrier and the sidebands with the resonance. The VCXO lock is called dispersion lock [12].

The interrogation signal produced by the electronic package, which is in the exponent form for simple expression, is then

$$
E_{i n c}=E_{0} \exp \left[i\left(\omega t+m \sin \omega_{m} t\right)\right]
$$

where $E_{0}$ is the amplitude of the interrogation signal, $\omega$ and $\omega_{m}$ are the carrier angular frequency and the mo- dulation angular frequency respectively and $m$ is the modulation index [13]. Assuming $E_{0}=1$, this expression is expanded, using Bessel functions [14,15], to obtain

$$
\begin{gathered}
E_{\text {int }}= \\
{\left[\begin{array}{c}
\left.J_{0}(m)+\sum_{n=1}^{\infty} 2(i)^{n} J_{1}(m) \sin \left(\omega_{m} t\right)\right] \exp (i \omega t) \approx \\
{\left[J_{0}(m)+2 i J_{1}(m) \sin \left(\omega_{m} t\right)\right] \exp (i \omega t)=} \\
J_{0}(m) \exp (i \omega t)+J_{1}(m)\left\{\exp \left[i\left(\omega+\omega_{m} t\right)\right]-\right. \\
\left.\exp \left[i\left(\omega-\omega_{m} t\right)\right]\right\} .
\end{array}\right.}
\end{gathered}
$$

In this case, it is sufficient to take into account only the carrier and the first-order side bands determined by $J_{0}(m)$ and $J_{1}(m)$ respectively, because the Bessel functions of higher orders become very small $[16,17]$.

The frequency response of the discriminator can be written as

$$
H(i \omega)=U(\omega)+i V(\omega) .
$$

$U(\omega)$ and $V(\omega)$ are the real part and the imaginary part of the imaginary part of the discriminator response, having angular frequency $\omega_{0}$ and linewidth $\gamma$.

The output signal becomes

$$
\begin{gathered}
E_{\text {out }} \approx J_{0}(m)[U(\omega)+i V(\omega)] \exp (i \omega t)+ \\
J_{1}(m)\left[U\left(\omega+\omega_{m}\right)+i V\left(\omega+\omega_{m}\right)\right] \times
\end{gathered}
$$




$$
\begin{gathered}
\exp \left[i\left(\omega+\omega_{m}\right) t\right]-J_{1}(m)\left[U\left(\omega-\omega_{m}\right)+\right. \\
\left.i V\left(\omega-\omega_{m}\right)\right] \exp \left[i\left(\omega-\omega_{m}\right) t\right] .
\end{gathered}
$$

If there is no error signal $\omega=\omega_{0}$, the signal, after the envelope detector and the filter at frequency $\omega_{m}$, which is obtained through multiplying the output signal by its complex conjugate and retaining the $\omega_{m}$ terms, is found to be

$$
\begin{gathered}
S\left(\omega_{0}, \omega_{m}\right)=2 \times J_{0}(m) J_{1}(m) \times \\
{\left[\varepsilon\left(\omega_{0}, \omega_{m}\right) \cos \left(\omega_{m} t\right)+\lambda\left(\omega_{0}, \omega_{m}\right) \sin \left(\omega_{m} t\right)\right]} \\
\varepsilon\left(\omega_{0}, \omega_{m}\right)=U\left(\omega_{0}\right) \times\left[U\left(\omega_{0}+\omega_{m}\right)-U\left(\omega_{0}-\omega_{m}\right)\right]+ \\
V\left(\omega_{0}\right) \times\left[V\left(\omega_{0}+\omega_{m}\right)-V\left(\omega_{0}-\omega_{m}\right)\right] \\
\lambda\left(\omega_{0}, \omega_{m}\right)=U\left(\omega_{0}\right) \times\left[V\left(\omega_{0}+\omega_{m}\right)+V\left(\omega_{0}-\omega_{m}\right)\right]- \\
V\left(\omega_{0}\right) \times\left[U\left(\omega_{0}+\omega_{m}\right)+U\left(\omega_{0}-\omega_{m}\right)\right] .
\end{gathered}
$$

Assuming the difference value between the carrier angular frequency of the interrogation signal and the discriminator resonance angular frequency $x=\omega-\omega_{0}$ [18], the error signal is

$$
\begin{gathered}
\xi\left(x, \omega_{m}\right)=2 \times J_{0}(m) J_{1}(m) \times \\
{\left[\varepsilon\left(x, \omega_{m}\right) \cos \left(\omega_{m} t\right)+\lambda\left(x, \omega_{m}\right) \sin \left(\omega_{m} t\right)\right] .}
\end{gathered}
$$

In the absorption lock, the modulation frequency is much less to the resonance width $\gamma_{a}$ and

$$
\arctan \left[\varepsilon\left(x, \omega_{m}\right) / \lambda\left(x, \omega_{m}\right)\right] \approx 90^{\circ} .
$$

In this regime, only the cosine term in (6) plays the dominant role and it is called absorption spectrum curve. The normalized expression is

$$
\begin{aligned}
\xi_{a b s}\left(x, \omega_{m}\right) & =U(x) \times\left[U\left(x+\omega_{m}\right)-U\left(x-\omega_{m}\right)\right]+ \\
V(x) & \times\left[V\left(x+\omega_{m}\right)-V\left(x-\omega_{m}\right)\right] .
\end{aligned}
$$

In the same way of the dispersion lock, the modulation frequency is much more to the resonance width $\gamma_{d}$ and

$$
\arctan \left[\varepsilon\left(x, \omega_{m}\right) / \lambda\left(x, \omega_{m}\right)\right] \approx 0^{\circ} .
$$

Only the sine term in (6) survives, which is called dispersion spectrum curve [19-21] and becomes

$$
\begin{gathered}
\xi_{d i s}\left(x, \omega_{m}\right)=U(x) \times\left[V\left(x+\omega_{m}\right)+V\left(x-\omega_{m}\right)\right]- \\
V(x) \times\left[U\left(x+\omega_{m}\right)+U\left(x-\omega_{m}\right)\right] .
\end{gathered}
$$

\subsection{Error signals in passive hydrogen maser}

In the passive hydrogen maser, a simple mathematical analysis of the error signal can be made using the absorption and dispersion lock method. After the signal produced by the VCXO has passed through the frequency multiplier, divider, mixer and frequency modulator, the center frequency of the interrogation signal with frequency modulation approximates to the hydrogen hyperfine transition frequency $1.420405751 \mathrm{GHz}$ [22]. For simplicity, only the carrier and the first sidebands of the interrogation signal are considered. The interrogation signal enters the physical package as the excitation signal for the hydrogen transition and comes out with the frequency information of the cavity and hydrogen line [23].

The physical package of the passive hydrogen maser performs the function of a very narrow-band microwave amplifier and a frequency discriminator, produced by the hydrogen hyperfine transition and microwave cavity [24]. The general idea of absorption and dispersion lock can be applied to the passive hydrogen maser. However, the simple response function of a frequency discriminator cannot be used. A quantum mechanical calculation is needed. The frequency response of the cavity and hydrogen $[25,26]$ is

$$
\begin{aligned}
& H_{C}(i \omega)=H_{C R}(\omega)+i \times H_{C I}(\omega)= \\
& \frac{\gamma_{C}^{2}}{\gamma_{C}^{2}+\left(\omega-\omega_{0}\right)^{2}}+i \frac{\gamma_{C} \times\left(\omega-\omega_{0}\right)}{\gamma_{C}^{2}+\left(\omega-\omega_{0}\right)^{2}} .
\end{aligned}
$$

And

$$
\begin{gathered}
H_{H}(i \omega)=H_{H R}(\omega)+i \times H_{H I}(\omega) \\
H_{H R}(\omega)=\frac{\gamma_{H}^{2}(1-z)+\left(\omega-\omega_{0}\right)\left[\omega-\omega_{0}+\frac{\gamma_{H}}{\gamma_{C}}\left(\omega-\omega_{C}\right)\right]}{\left[\gamma_{H}(1-z)\right]^{2}+\left[\omega-\omega_{0}+\frac{\gamma_{H}}{\gamma_{C}}\left(\omega-\omega_{C}\right)\right]^{2}} \\
H_{H I}(\omega)=\frac{\gamma_{H}\left[\omega-\omega_{0}+\frac{\gamma_{H}}{\gamma_{C}}\left(\omega-\omega_{C}\right)\right]}{\left[\gamma_{H}(1-z)\right]^{2}+\left[\omega-\omega_{0}+\frac{\gamma_{H}}{\gamma_{C}}\left(\omega-\omega_{C}\right)\right]^{2}}
\end{gathered}
$$

where $\omega_{C}$ is the resonant frequency of the microwave cavity, which influences the hydrogen transition and has been called cavity pulling effect. As we discuss the VCXO loop, assume that the cavity error signal is neglected and $\omega-\omega_{C}=0 . z$ has the meaning of a normalized population difference, $z=1$ corresponds to the oscillation limit and $z<1$ to the passive maser operation, so it can be set $z=0.4$.

From the frequency response of the cavity, the error signal of the cavity is

$$
\begin{gathered}
E_{C}(x, t)=2 \times J_{0}(m) J_{1}(m) \times \\
{\left[\varepsilon_{C}\left(x, \omega_{m}\right) \cos \left(\omega_{m} t\right)+\lambda_{C}\left(x, \omega_{m}\right) \sin \left(\omega_{m} t\right)\right]}
\end{gathered}
$$


$\varepsilon_{C}\left(x, \omega_{m}\right)=H_{C R}(x) \times\left[H_{C R}\left(x+\omega_{m}\right)-H_{C R}\left(x-\omega_{m}\right)\right]+$

$$
\begin{gathered}
H_{C I}(x) \times\left[H_{C I}\left(x+\omega_{m}\right)-H_{C I}\left(x-\omega_{m}\right)\right] \\
\lambda_{C}\left(x, \omega_{m}\right)=H_{C R}(x) \times\left[H_{C I}\left(x+\omega_{m}\right)+H_{C I}\left(x-\omega_{m}\right)\right]- \\
H_{C I}(x) \times\left[H_{C R}\left(x+\omega_{m}\right)+H_{C R}\left(x-\omega_{m}\right)\right] .
\end{gathered}
$$

The error signal of the hydrogen is

$$
\begin{gathered}
E_{H}(x, t)=2 \times J_{0}(m) J_{1}(m) \times \\
{\left[\varepsilon_{H}\left(x, \omega_{m}\right) \cos \left(\omega_{m} t\right)+\lambda_{H}\left(x, \omega_{m}\right) \sin \left(\omega_{m} t\right)\right]} \\
\varepsilon_{H}\left(x, \omega_{m}\right)=H_{H R}(x) \times\left[H_{H R}\left(x+\omega_{m}\right)-H_{H R}\left(x-\omega_{m}\right)\right]+ \\
H_{H I}(x) \times\left[H_{H I}\left(x+\omega_{m}\right)-H_{H I}\left(x-\omega_{m}\right)\right] \\
\lambda_{H}\left(x, \omega_{m}\right)=H_{H R}(x) \times\left[H_{H I}\left(x+\omega_{m}\right)+H_{H I}\left(x-\omega_{m}\right)\right]- \\
H_{H I}(x) \times\left[H_{H R}\left(x+\omega_{m}\right)+H_{H R}\left(x-\omega_{m}\right)\right] . \quad(12)
\end{gathered}
$$

For the convenience of simulation, assume that $\omega_{m}=$ $100 \mathrm{~Hz}, \gamma_{C}=4000 \mathrm{~Hz}$ and $\gamma_{H}=4 \mathrm{~Hz}$ are plugged into (11) and (12) respectively and obtain the absorption and dispersion spectrum curves, as shown in Fig. 2. They clearly show that the absorption spectrum curve is the main component in the absorption lock with $\gamma \gg \omega_{m}$, while the dispersion spectrum curve is the main component in the dispersion lock with $\gamma \ll \omega_{m}$. Because of the antisymmetric for frequency difference of the spectrum curves, they are able to be used for discriminating frequency [27]. Although the resonance linewidth in dispersion lock is narrow, such as the hydrogen natural linewidth $\gamma_{H} \approx 2 \mathrm{~Hz}$, the frequency range of the discriminator is from $-\omega_{m}$ to $\omega_{m}$, which adopts the frequency modulation spectroscopy and modulation transfer spectroscopy technology [28].

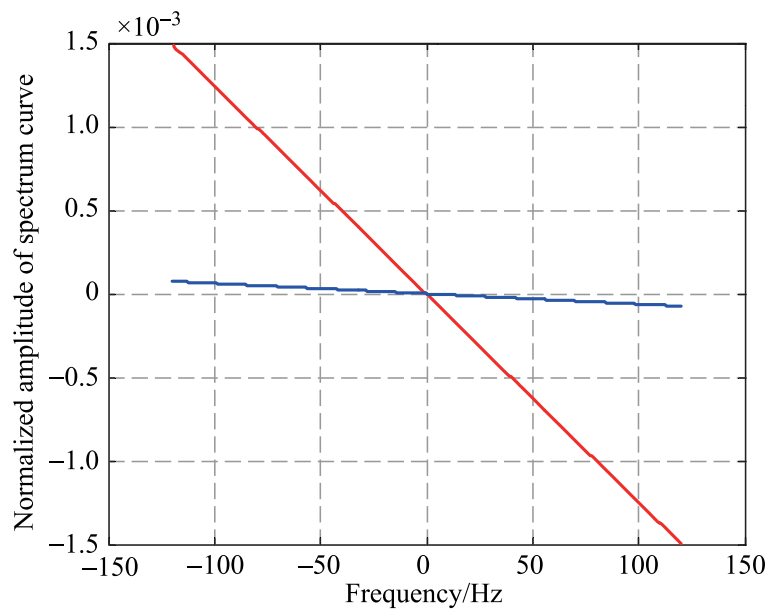

(a) The resonance width $\gamma=4000 \mathrm{~Hz} \gg>\omega_{m}=100 \mathrm{~Hz}$

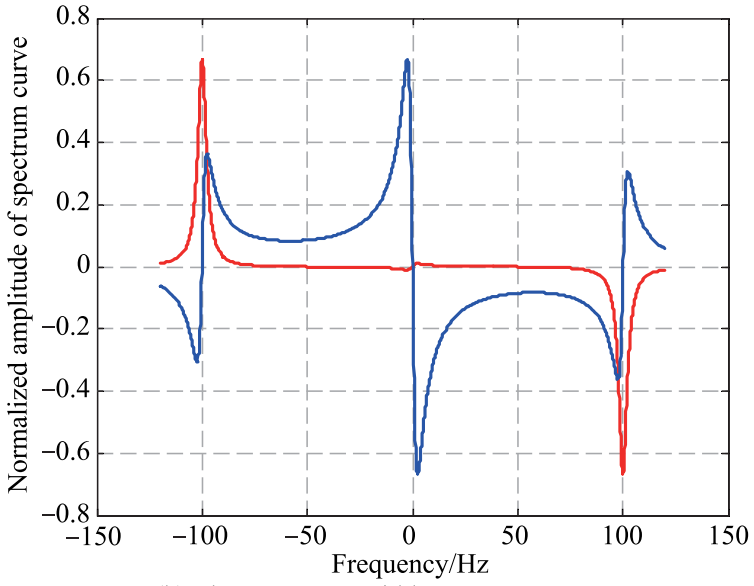

(b) The resonance width $\gamma=4 \mathrm{~Hz}<<\omega_{m}=100 \mathrm{~Hz}$

: Absorption spectrum curve;

: Dispersion spectrum curve.

Fig. 2 Absorption and dispersion spectrum curves of different linewidths

The typical parameter values of the passive hydrogen maser are as follows : $\omega_{0}=1420405751 \mathrm{~Hz}, \omega_{m}=$ $12500 \mathrm{~Hz}, \gamma_{C}=180000 \mathrm{~Hz}, \gamma_{H}=2 \mathrm{~Hz}$. The phase angle of the error signal, for near tuning condition and referred to the frequency modulation signal $\sin \left(\omega_{m} t\right)$, is given by

$$
\theta_{C}=\arctan \frac{\varepsilon_{C}\left(x, \omega_{m}\right)}{\lambda_{C}\left(x, \omega_{m}\right)} \approx \arctan (7.1653) \approx 82.06^{\circ} .
$$

And the phase angle of the hydrogen error signal is given by

$$
\begin{gathered}
\theta_{H}=\arctan \frac{\varepsilon_{H}\left(x, \omega_{m}\right)}{\lambda_{H}\left(x, \omega_{m}\right)} \approx \arctan \left(-6.4 \times 10^{-5}\right) \approx \\
-0.0037^{\circ} .
\end{gathered}
$$

The phase difference between the two phase angles approaches $90^{\circ}$. With $\sin \left(\omega_{m} t\right)$ being as the reference phase direction and perpendicular to $\cos \left(\omega_{m} t\right)$, the result of the vector model is shown in Fig. 3.

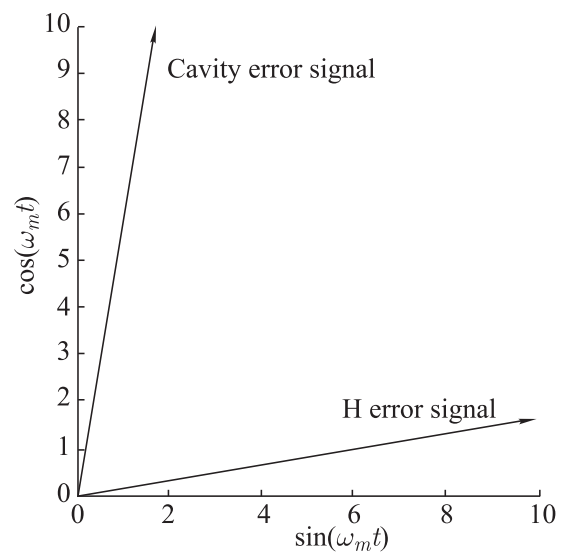

Fig. 3 Vectors sketch diagram of the cavity phase angle and hydrogen phase angle 


\subsection{Separation of the two error signals}

As the passive hydrogen maser is based on the single frequency modulation approach, it requires a proper phase selection of the synchronous detectors presented in the VCXO control loop and in the cavity control loop, in order to separate the two error signals. Conventionally, the phase is first adjusted for obtaining a maximum hydrogen error signal in the VCXO loop, for nearly tuned cavity, in a second step the reference phase of the cavity loop synchronous detector is shifted resulting in a maximum cavity error signal in the cavity loop. They are $\theta_{H}$ and $\theta_{C}$ in Fig. 3 and the phase selection in the VCXO loop is shown in Fig. 4(a). In this way, the maximum error signal can be obtained in each loop, while one is actually contaminated by the presence of the other error signal, because of the imperfect verticality of the two phase angles are not perfectly vertical.

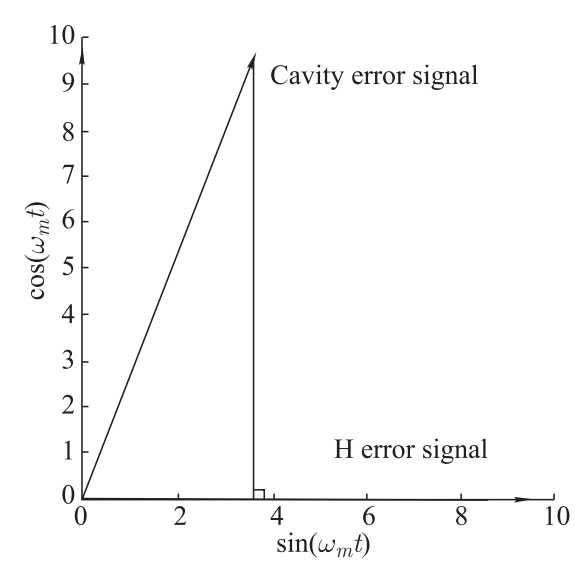

(a) The traditional method

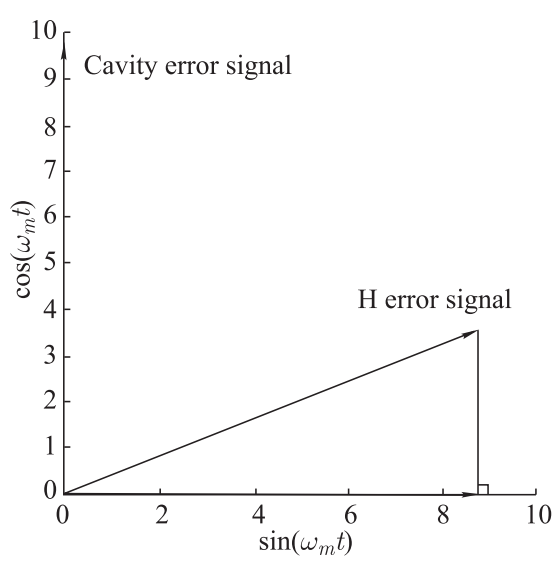

(b) The new method

Fig. 4 Schematic diagram of the phase angle selection in VCXO loop

The theoretical calculation shows that a specific phase angle exists, on which one loop signal is entirely separate from the other, i.e. the VCXO loop error signal and the cavity loop error signal do not contain the component of each other. For the VCXO loop, if the phase angle is perpendicularity to the vector of the cavity error signal, there will be no cavity error signal while the $\mathrm{H}$ error signal amplitude is reduced, as shown in Fig. 4(b). The theory for the cavity loop is the same as the VCXO loop.

\section{Matlab simulation of separation}

The diagram of Fig. 5 is a good starting point to illustrate the application of the new phase selection with the independence of two error signals. The simulation is performed with the Matlab 2010 [29]. The H error signal is a sine wave with the phase $\theta_{H}=-0.0037^{\circ}$ and the cavity error signal with the phase $\theta_{C}=82.06^{\circ}$ in the simulation. The summation of the two error signals is separated by two synchronizing square waves signals with different phases. For simplicity, the simulation time is $10 \mathrm{~s}$ and the sine wave frequency is $1 \mathrm{~Hz}$.

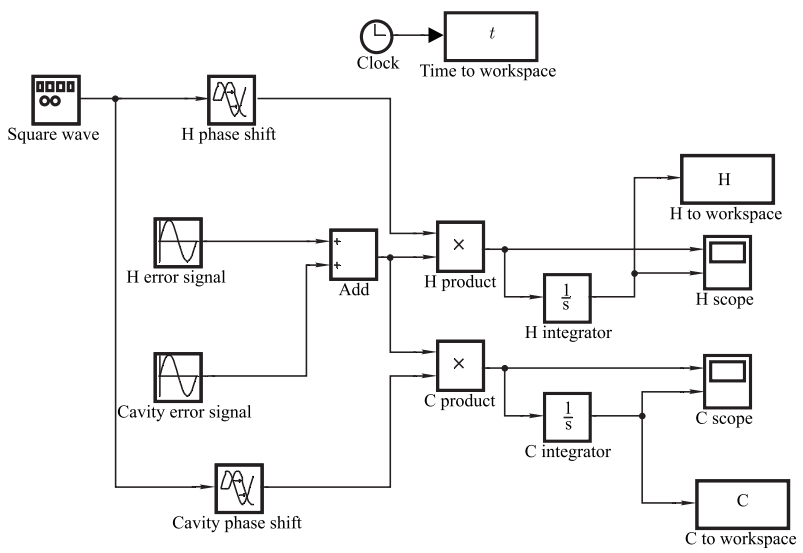

Fig. 5 Two error signals separation simulation

The traditional method of the separation is keeping the phases of the synchronizing square wave the same as the two error signals respectively. The error signals are shown in Fig. 6. In this way, the error signals after the integrator change with different amplitudes of the other error signal as shown in Fig. 7 and Fig. 8. If the new phase selections are set in the simulation model, the error signals are the same with different amplitudes of the other error signal as shown in Fig. 9 and Fig. 10. The simulation clearly shows that the two error signals are independent in the new phase selections.

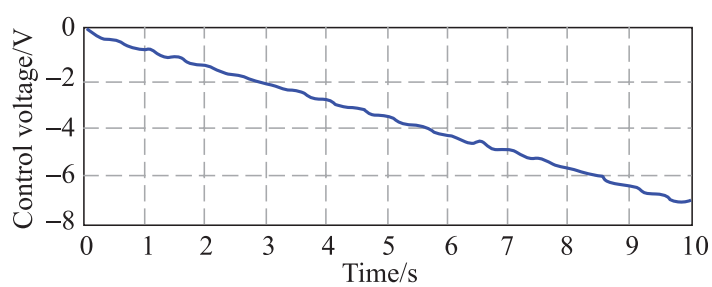

(a) H error signal 


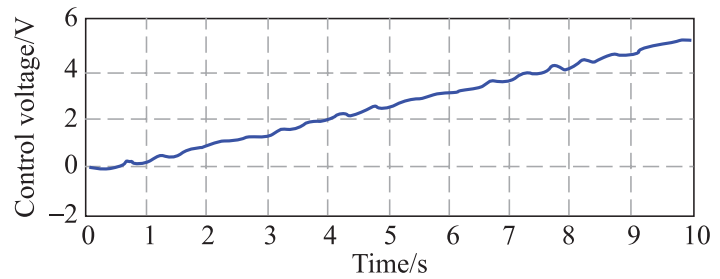

(b) Cavity error signal

Fig. 6 Matlab simulation of the two error signals

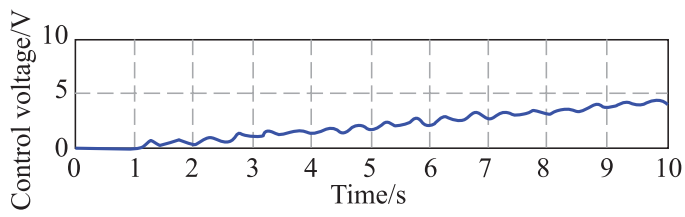

(a) $1 \mathrm{~V} \mathrm{H}$ error signal

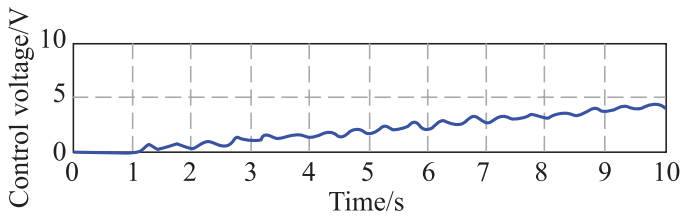

(b) $2 \mathrm{~V} \mathrm{H}$ error signal

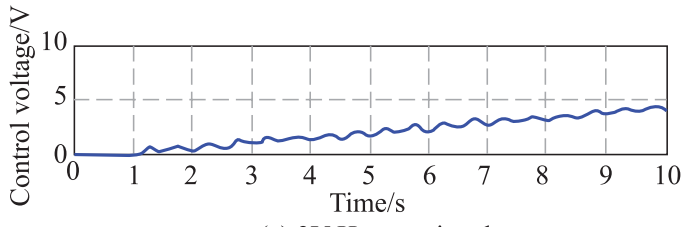

(c) $3 \mathrm{~V} \mathrm{H}$ error signal

Fig. 7 Cavity error signals with different $\mathrm{H}$ error signals in traditional method

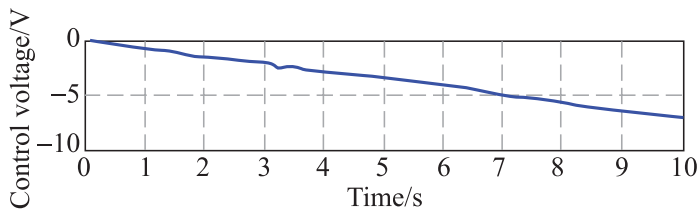

(a) $1 \mathrm{~V}$ cavity error signal

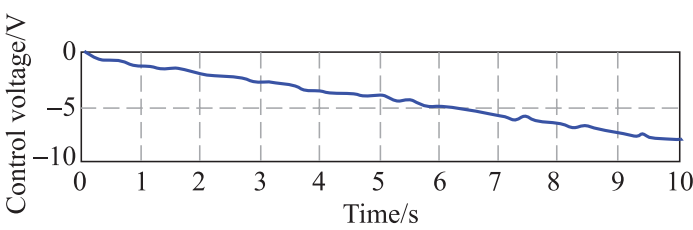

(b) $2 \mathrm{~V}$ cavity error signal

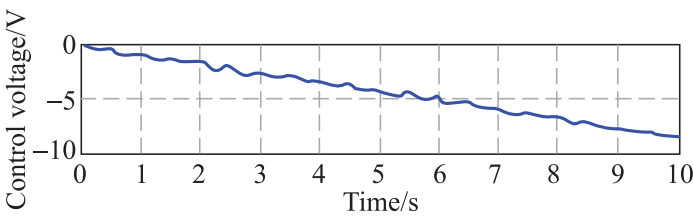

(c) $3 \mathrm{~V}$ cavity error signal

Fig. $8 \mathrm{H}$ error signals with different cavity error signals in traditional method

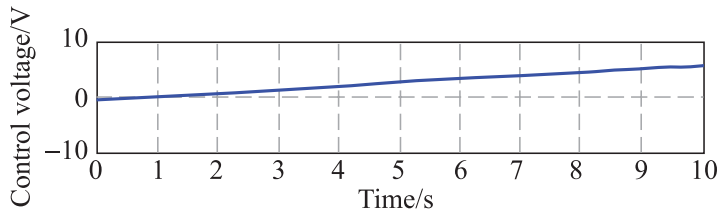

(a) $1 \mathrm{~V} \mathrm{H}$ error signal

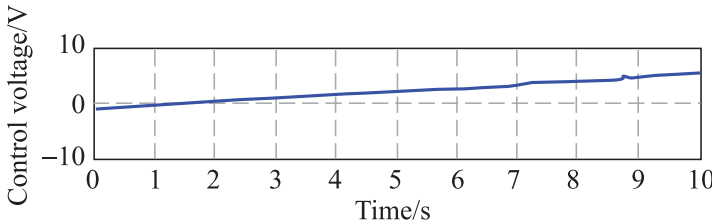

(b) $2 \mathrm{~V} \mathrm{H}$ error signal

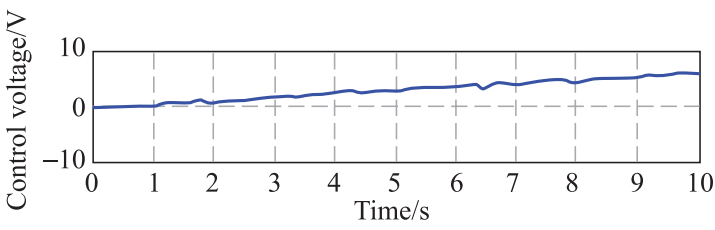

(c) $3 \mathrm{~V} \mathrm{H}$ error signal

Fig. 9 Cavity error signals with different $\mathrm{H}$ error signals in new phase selections

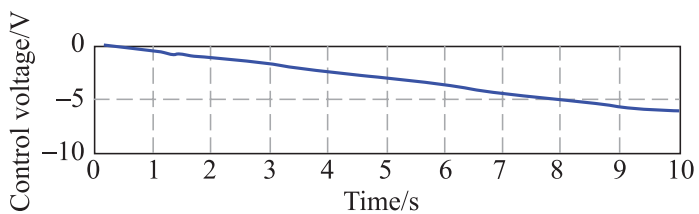

(a) $1 \mathrm{~V}$ cavity error signal

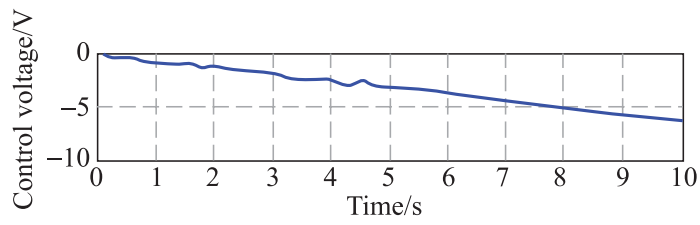

(b) $2 \mathrm{~V}$ cavity error signal

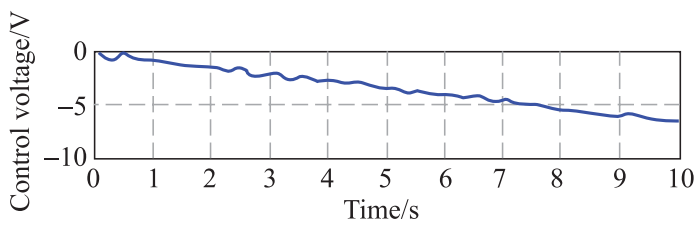

(c) $3 \mathrm{~V}$ cavity error signal

Fig. $10 \mathrm{H}$ error signals with different cavity error signals in new phase selections

\section{Experiment}

The experiment is to debug the demodulation and integral electrocircuit of the passive hydrogen maser in open loops. The signal from the physical package, with the error information, becomes IF signal through the LNA and down converter, and the error signals are processed by the demodulation and integral electrocircuit. In the experiment, a voltage source is used to supply voltage for VCXO and variable capacitance diode (VCD) with two independent 
channels to control the frequency of VCXO and cavity. In the traditional method, the phase selection is operated as follows. First, turn off the atom transition of maser, and the error signal is just from cavity. Second, adjust the phase of the error signal same with the square wave in the cavity loop, and then change the voltage of the VCD to minimize the cavity error signal. Finally, turn on the atom transition of maser and adjust the phase of the error signal, which is just from the hydrogen atom transition, same with the square wave in the VCXO loop, as shown in Fig. 11. Due to the out of strict vertical of the phases, the two error signals are not separated absolutely. When the voltage of the VCD changes, the error signal after interaction with the square wave in the VCXO loop is changed because of the influence from the cavity error signal.

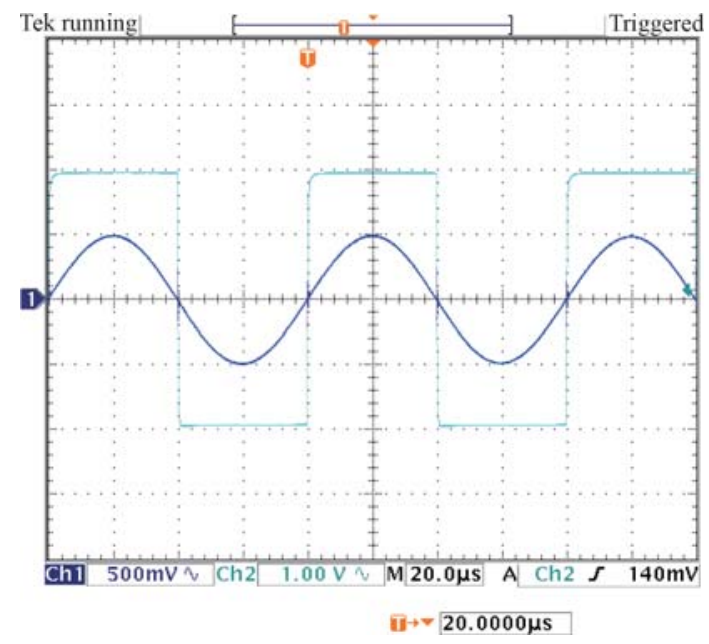

Fig. 11 Phase selection in traditional method

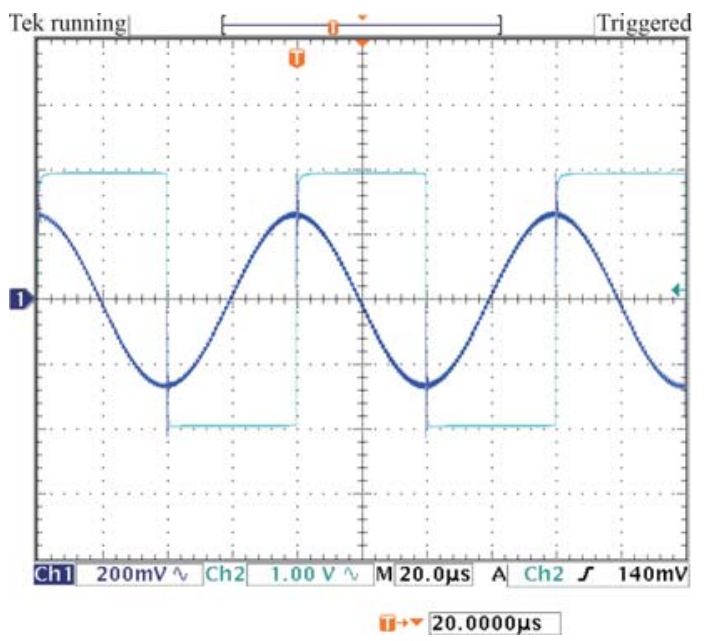

Fig. 12 New phase selection

The new phase selection is operated as follows. First, turn off the atom transition of maser and it is just the cavity error signal. Second, adjust the error signal and square wave with 90 degrees phase difference in the VCXO loop, and then minimize the cavity error signal. Finally, turn on the atom transition of maser and adjust the error signal and square wave with 90 degrees phase difference in the cavity loop, as shown in Fig. 12. The two error signals are independent in the two loops. When the voltage of the VCD changes, the error signal after interaction with the square wave in the VCXO loop is invariable.

\section{Conclusions}

The frequency modulation technology is a key technology of the passive hydrogen maser, which is one of the widely used frequency standards. The theoretical expression of the single frequency modulation for the passive hydrogen maser and the function of the cavity and $\mathrm{H}$ line error signals separation are derived, which are in good agreement with the test. The calculation results of the absorption and dispersion spectrum curves validate the frequency discrimination approaches of the cavity loop and the VCXO loop respectively. The traditional method of the two error signals separation is analyzed, and then it describes a new improved method for the passive hydrogen servo loops consisting in the use of a single modulation frequency and phase discrimination for the separation. There is interaction of the two error signals in the new selection of the phase setting, which is deduced theoretically and validated by the simulation. The preliminary experimental result confirms the feasibility of this new approach, which is conductive to improve significantly the long term performance of the passive hydrogen maser.

\section{References}

[1] P. Waller, F. Gonzalez. Long-term performance analysis of GIOVE clocks. Proc. of the 42nd Annual Precise Time and Time Interval Meeting, 2010: 171-179.

[2] W. M. Wang, G. H. Shen. Design and experimental study of the hydrogen source of space passive hydrogen maser. Proc. of the IEEE International Frequency Control Symposium, 2016: $1-4$.

[3] W. M. Wang. The current development of atomic hydrogen clock and the future technology development trends in China. Navigation Positioning and Timing, 2015, 2(6): 48-54. (in Chinese)

[4] J. Vanier, C. Audoin. The quantum physics of atomic frequency standards. Cleveland: Chemical Rubber Company Press, 1988.

[5] F. L. Walls, D. A. Howe. A passive hydrogen maser frequency standard. Proc. of the 32nd Annual Symposium on Frequency Control, 1978: $492-498$.

[6] C. F. Lin, T. X. Liu. Miniature passive hydrogen maser at Shanghai Observatory. Acta Metrologica Sinica, 2003, 24(1): 69-71. (in Chinese)

[7] Z. W. Lin, B. C. Qu. A single modulation frequency method used in the passive $\mathrm{H}$ maser. Annals of Shanghai Observatory Academia Sinica, 2009, 29(1): 122 - 127. (in Chinese) 
[8] D. Kleppner, H. C. Berg. Hydrogen-maser principles and techniques. Physical Review, 1965, 138(4A): A972 - A983.

[9] J. Li, J. H. Zhang. Space passive hydrogen maser. Proc. of the IEEE International Frequency Control Symposium, 2016: $5-9$.

[10] G. Busca, Q. Wang. Cavity pulling in Galileo passive hydrogen maser. Proc. of the IEEE International Frequency Control Symposium, 2003: 86-89.

[11] D. A. Howe, F. L. Walls. A small passively operated hydrogen maser. Proc. of the 33rd Annual Symposium on Frequency Control, 1979: 554-562.

[12] G. Busca, H. Brandenberger. Passive H maser. Proc. of the 33rd Annual Symposium on Frequency Control, 1979: $563-$ 568.

[13] Z. Y. Bi, L. E. Ding. Measuring reflection of optical cavity by optical heterodyne detection. Journal of East China Normal University (Natural Science), 1989, 3(1): 41 -46. (in Chinese)

[14] B. Y. Chen. Radio frequency communication circuit. Beijing: Science Publishing Company, 2006. (in Chinese)

[15] Y. P. Huang, L. Q. Liu. Digital communications technology. Beijing: Mechanical Industry Press, 2015. (in Chinese)

[16] J. C. Li, Y. H. Zhang. Application of frequency modulation in resonator fiber optic gyro. Journal of Shanghai Jiaotong University, 2000, 34(2): 226-228. (in Chinese)

[17] J. C. Li. Frequency modulation theory and emulation of resonator fiber optic gyro. Aerospace Shanghai, 2002, 2(1): $24-$ 26. (in Chinese)

[18] Y. X. Peng, W. B. Li. Theoretical calculation of modulation signal in iodine absorption laser frequency stabilization. Optical Technique, 2004, 30(1): 95 -97. (in Chinese)

[19] G. C. Bjorklund. Frequency-modulation spectroscopy: a new method for measuring weak absorptions and dispersions. $O p$ tical Society of America, 1980, 5(1): 15-17.

[20] J. Mandon, G. Guelachvili. Frequency-modulation Fourier transform spectroscopy: a broadband method for measuring weak absorptions and dispersions. Optical Letters, 2007, 32(15): $2206-2208$.

[21] E. D. Black. An introduction to Pound-Drever-Hall laser frequency stabilization. Physical Review, 2001, 69(1): 79-87.

[22] P. Berthoud, I. Pavlenko. The engineering modal of the space passive hydrogen maser for the European global navigation satellite system Galileo. Proc. of the IEEE International Frequency Control Symposium, 2003: 90-94.

[23] Q. H. Wang, P. Mosset. Verification and optimization of the physics parameters of the onboard Galileo passive hydrogen maser. Proc. of the 38th Annual Precise Time and Time Interval Meeting, 2006, 10: 1-13.

[24] M. Belloni, M. Gioia. Space mini passive hydrogen maser. Proc. of the IEEE International Frequency Control and the European Frequency and Time Forum, 2011: 1-5.

[25] J. H. Shirley. Dynamics of a simple maser model. American Journal of Physics, 1968, 36(11): $949-963$.

[26] J. Viennet, C. Audoin. Cavity pulling in passive frequency standards. IEEE Trans. on Instrumentation and Measurement, 1972, 21(3): 204-209.

[27] Y. Q. Wang, Q. J. Wang. Frequency standards theory. Beijing: Science Publishing Company, 1986. (in Chinese)

[28] Z. Y. Bi, J. X. Ding. Stabilization of mini-Nd:YVO4 laser us- ing I2 hyperfine transition. Chinese Science Bulletin, 2000, 45(22): 2461 -2464. (in Chinese)

[29] D. F. Zhang. Matlab Simulink modeling and simulation. Beijing: Publishing House of Electronics Industry, 2009. (in Chinese)

\section{Biographies}

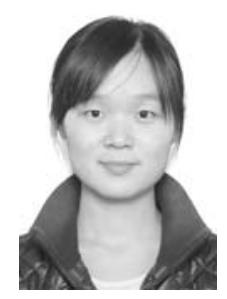

Hefei Zheng was born in 1991. She received her B.E. degree in electronic science and technology from Yanshan University in 2013. She is a Ph.D. candidate in The Second Academy of China Aerospace Science and Industry Corporation (CASIC) and is engaged in atomic clock research in the Atomic Frequency Standard Laboratory in Beijing Institute of Radio Metrology and Measurement. Her research interests are space and ground hydrogen maser.

E-mail: zhenghefei1@ sina.com

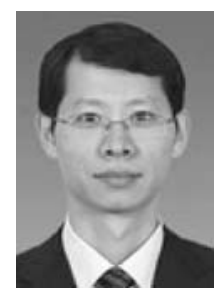

Jing Li was born in 1972. He received his B.E. degree in electronic instrument and measurement from Harbin Institute of Technology in 1995. He is currently a senior engineer in the Atomic Frequency Standard Laboratory in Beijing Institute of Radio Metrology and Measurement. His research interests focus on the circuit design and system design of active and passive hydrogen maser.

E-mail: lijing721217@ hotmail.com

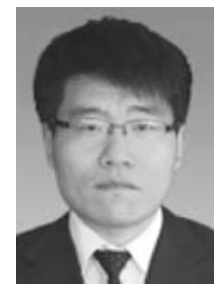

Wenming Wang was born in 1985. He received his Ph.D. degree in science from Shanghai Astronomical Observatory in 2003. He is currently an intermediate engineer in the Atomic Frequency Standard Laboratory in Beijing Institute of Radio Metrology and Measurement. His research interests focus on development of active hydrogen maser and research of space passive hydrogen maser.

E-mail: wwm_kingdom@163.com

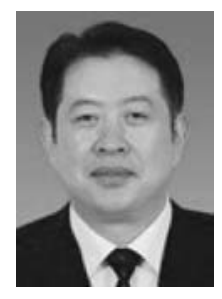

Lianshan Gao was born in 1965. He received his B.E. degree in 1986. He is currently a researcher in Beijing Institute of Radio Metrology and Measurement and the director of the Atomic Frequency Standard Laboratory. His research interests focus on the atomic clock technology.

E-mail: gaolianshan258@ sohu.com

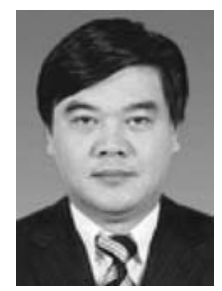

Keming Feng was born in 1966. He received his M.S. degree in microwave technique from Chengdu University of Electronic Science and Technology in 1990. He is currently a researcher and the director of Beijing Institute of Radio Metrology and Measurement. He is an important contribution expert in CASIC and is granted a special allowance from government. His research interests focus on the atomic clock technology.

E-mail: kmfeng@sina.com 